\title{
Der Altar des unbekannten Gottes.
}

Von P. Corssen in Berlin-Dahlem.

In seinen ausgezeichneten Untersuchungen zur Formengeschichte religiöser Rede hat E. Norden bekanntlich die Ansicht entwickelt, Lukas - ich gebrauche den herkömmlichen Namen der Kürze halber hier und im folgenden, ohne Verbindlichkeit - habe in der Areopagrede eine Stelle aus einer von Apollonios von Tyana in Athen gehaltenen Rede benutzt. Der Passus des Philostratos, auf den es dabei ankommt, hat bei A. Harnack in den Texten und Untersuchungen XXXIX 1,1913, eine wesentlich andere Auslegung erfahren, gegen die sich wiederum Reitzenstein in den Neuen Jahrbüchern für das klass. Altert. XXXI 6,1913, S. $393 \mathrm{ff}$. mit großer Lebhaftigkeit gewendet hat.

Ich gestehe, daß ich zu denjenigen gehöre, die durchaus im Banne der Nordenschen Interpretation standen, und $\mathrm{daB}$ ich darin durch Reitzenstein noch-bestärkt wurde, bis ich vor einigen Tagen durch den Einwurf eines sehr besonnen und kühl urteilenden Kollegen von mir stutzig wurde. Es ist zu erwarten, daß dieselben Bedenken, die meinem Kollegen und mir gekommen sind, auch andern kommen werden, und mancher mag dadurch bewogen werden, Nordens Kombinationen überhaupt aufzugeben, weil sie in einem einzelnen Punkte nicht stimmen. Allein ein Bau braucht darum noch nicht abgetragen zu werden, weil einer der Pfeiler, auf denen er ruht, sich nicht als zuverlässig erweist. Im Interesse des Baumeisters und seines Werkes aber können die Grundlagen, die er gelegt hat, nicht sorgfältig genug untersucht werden, und wenn sie mit Schäden behaftet sind, ist es besser, sie so bald wie möglich klarzulegen und auszubessern.

Ich will daher die Stelle in der Schrift des Philostratos, die den Ausgangspunkt von Nordens Untersuchung bildet, zunächst ohne alle Nebenrücksichten lediglich aus ihrem eigenen Zusammenhang interpretieren, um zu erkennen, ob die Wendung, um die der Kampf entbrannt

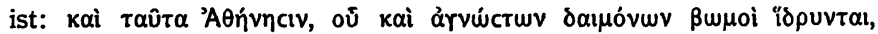
mit dem Grundgedanken der Stelle natürlich zusammenhängt oder ihr willkürlich eingefügt ist. 
Die Geschichte, die Philostratos VI 3 dem von ihm so ausgiebig benutzten Damis Wort für Wort nacherzählt, betrifft einen Jüngling aus Naukratis, Timasion, den die Reisenden am Nil treffen ( $\mu \in \mathbf{p} \alpha$ ḱı

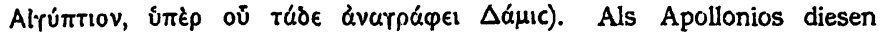
kommen sicht, den er zuvor nie gesehen und von dem er nie etwas gehört hatte, erzählt er den Gefährten sein Schicksal, indem er mit

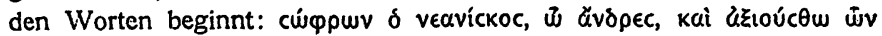
deîta. Die Geschichte selbst hatte Damis mit wenigen Worten voraufgeschickt. Es handelt sich in ihr darum, daß der Jüngling seine

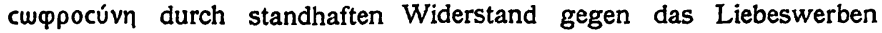
einer. Stiefmutter bewiesen hatte. Gleich von vornherein wird die 'Stiefmutter mit der Phädra in Parallele gestellt und dadurch eine Vergleichung zwischen Timasion und Hippolytos vorbereitet, die hinterher von Apollonios zum Lobe des Timasion durchgeführt wird. Nachdem nämlich dieser näher gekommen war und seinen Wunsch, an der Fahrt der Philosophen teilzunehmen, ausgesprochen hatte, erzählt er, auf Apollonios' Aufforderung, nach einigem Zögern und mit großer Bescheidenheit selbst seine Geschichte, in genauer Übereinstimmung mit dem, was die Hörer soeben von Apollonios vernommen hatten. Hierauf

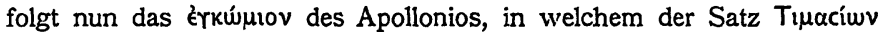

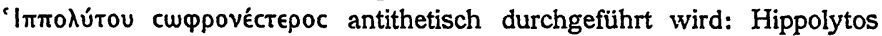
schmähte die Aphrodite und wollte von der Liebe nichts wissen, Timasion aber, der täglich zur Aphrodite betet, dient ihr und erlag doch nicht der Liebenden, weil er aus Respekt vor der Göttin sich vor un-

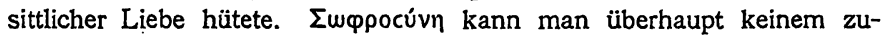
erkennen, der mit irgendeiner Gottheit verfeindet ist, wie Hippolytos

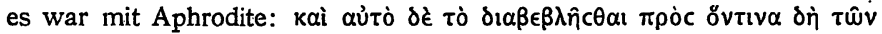

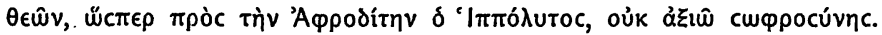

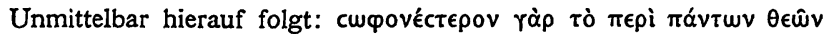

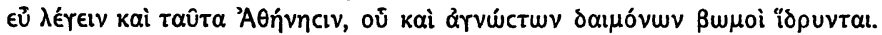

Der Gedanke hat hier einen allgemeinen Ausdruck angenommen - $\epsilon \hat{U} \lambda \lambda^{\prime} \gamma \epsilon \iota v$ ohne bestimmtes Subjekt - was aber schon im vorhergehenden vorbereitet war, wo die Anwendung des zunächst allgemein

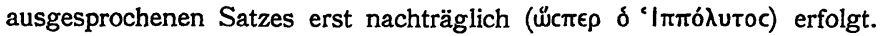
Aber der Komparativ cwppovéctєpov deutet an, daß die Vergleichung fortgesetzt wird. Hinter der allgemeinen Form verbergen sich also zwei Gedanken: Timasion redet von allen Göttern gut und verdient

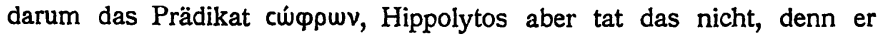
schmähte ja die Aphrodite, und hat also weniger Anspruch darauf.

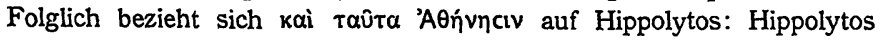


hätte um so mehr Grund gehabt, auch der Aphrodite Respekt zu beweisen, weil man in Athen sogar unbekannten Göttern Altäre errichtet hat, wohingegen Timasion seine Heimat Naukratis eine solche besondere Aufforderung zur Frömmigkeit nicht bot, was zwar nicht ausdrücklich ausgesprochen wird, aber sich aus dem Zusammenhang von selbst ergibt. Gleichwohl nennt Apollonios den Jüngling Hippolytos, weil beide in der Hauptsache übereinstimmen, nämlich in ihrem Verhalten gegen ihre Stiefmutter. Der Jüngling ist dem Hippolytos auch in körperlicher Bildung ähnlich, aber er übertrifft ihn wiederum auch

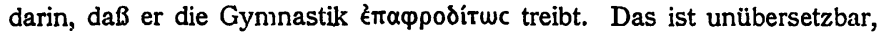

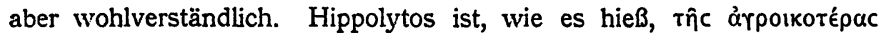

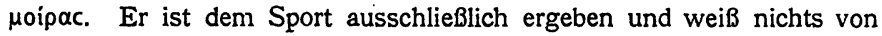
veredelnder Liebe. Der Naukratite aber, der zugleich im Dienst der Aphrodite steht, verbindet mit der Gymnastik edle Liebensıürdigkeit und feine Bildung.

So wird die Vergleichung des Timasion mit Hippolytos bis zum

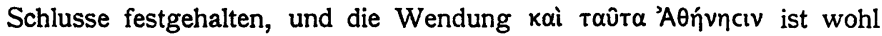
überraschend, aber sie widerspricht nicht dem thema probandum, zu dessen Unterstützung sie dient. Wir haben es mit einem rein akademischen Thema-zu tun, das ganz rhetorisch durchgeführt ist, ohne Rücksicht auf Raum und Zeit. $\mathrm{Ob}$ es am Nil oder am Ganges, in Rom oder Athen behandelt wird, ist dabei ganz gleichgültig. Daß die Beziehungen des Hippolytos zu Troezen ignoriert werden, darf nicht auffallen. Wurde doch das Grabmal des Theseussohnes so gut in Athen wie in Troezen gezeigt (Pausan. I 22, 1.2).

Man kann daher nicht sagen, die ganze Geschichte sei auf das athenische Kultuskuriosum angelegt (Norden S. 42). Die Geschichte ist vielmehr darauf angelegt, mit allen Mitteln die cwppocúvn des Naukratiten ins Licht zu stellen. Dazu muß auch das athenische Kultuskuriosum dienen.

Auf dem Wege, den Norden eingeschlagen hat und auf dem ihm Reitzenstein gefolgt ist, läßt sich daher nicht beweisen, daß dieses Kuriosum einem anderen Zusammenhang entstammen müsse. Es ist richtig, daß Hippolytos als Kontrastfigur zu dem Naukratiten herangeholt ist, aber die Bemerkung über den Altar der unbekannten Götter ist mit ebendieser Kontrastfigur verknüpft. Nicht durch diese Bemerkung ist die Kontrastfigur bedingt, sondern umgekehrt ist die Bemerkung durch die Kontrastfigur hervorgerufen.

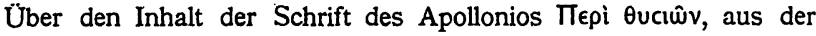
nach Nordens Meinung (S. 43) der Satz über die unbekannten Götter 
entlehnt ist, hat Philostratos sich an zwei Stellen ausgesprochen, III 41 und IV 19. Es waren in dem Buche Anweisungen gegeben, wie man einem jeden Gotte in angemessener und ihm wohlgefälliger Weise opfern könne (III 41). Diese Anweisungen aber gingen derartig ins einzelne, daß genau für jeden einzelnen Gott die Tages- und Nachtzeit angegeben war, $z u$ der man ihm $z u$ opfern, spenden oder $z u$ ihm $z u$ beten habe, wie auch der angemessene Ort dafür (IV 19). Daß aber das Buch darüber hinausging und sich zu einer höheren Auffassung erhob, zeigt das bei Eusebios Pr. ev. IV 13 erhaltene, von Norden S. 39 angeführte, wörtliche Zitat.

Philostrat sagt, er habe dieses Buch in vielen Heiligtümern, Städten und Häusern weiser Männer gefunden (III 41). Damis behauptete, es

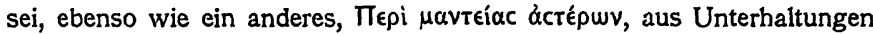
mit dem Inder Iarchas hervorgegangen (ebenda). Das ist natürlich eine Fiktion, aber eine Fiktion, die um so eher gewagt werden konnte, wenn das Buch rein systematisch aufgebaut war und jede Einkleidung oder Anknüpfung an irgendein wirkliches oder vorgebliches Faktum fehlte.

Damis erzählte auch, daß Apollonios in Athen sehr viele Vorträge gehalten habe, von denen er, Damis, aber nur diejenigen aufgezeichnet habe, in denen wirklich bedeutende Dinge behandelt worden seien. Der erste dieser Vorträge habe von Opfern gehandelt. Hierauf folgt eine genauere Inhaltsangabe, die der Schrift des Apollonios $\Pi \epsilon \rho i$ Guciŵv entspricht, wie aus dem Zusatz des Philostratos hervorgeht, es gebe auch ein Buch, in welchem Apollonios diesen Gegenstand behandle.

Damis hatte also in seinen Roman auch einen angeblich von Apollonios in Athen über Opfer gehaltenen Vortrag verflochten, der wesentlich auf der bekannten Schrift des Apollonios beruhte. Philostrat läßt auch noch genau erkennen, wie der Vortrag eingekleidet war. Apollonios war von dem Hierophanten die Beteiligung an den Epidaurien ${ }^{1}$ verweigert worden mit der Begründung, er würde keinen Gaukler weihen und einem kultisch Unreinen nicht Eleusis öffnen ( $\mu \grave{\eta}$ rà $\rho$ ăv

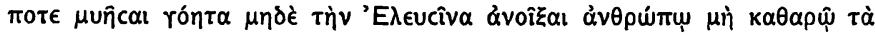

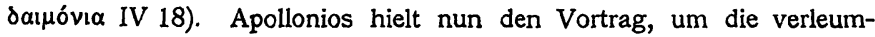
derischen und unwissenden Bemerkungen des Hierophanten über ihn durch die Darlegung einer genauen Kenntnis des Opferwesens zu widerlegen, wobei er an die Opferfreudigkeit der Athener anknüpfte. ${ }^{2}$

1 Vgl. A. Mommsen, Feste der Stadt Athen, 1898, S. $216 \mathrm{f}$.

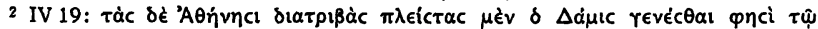

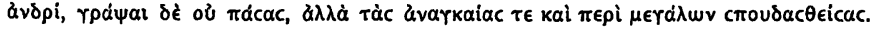

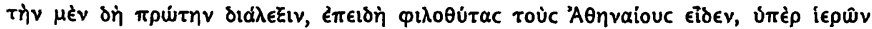


Die Uberlieferung, die wir haben, ergibt also nicht, daß Apollonios einen Vortrag über das Opferwesen in Athen gehalten, sondern daß Damis in seinem Roman dies fingiert hat. Norden hat demnach seine Hypothese auf eine unerweisliche Voraussetzung gegründet. Sollen wir darum über sie zur Tagesordnung übergehen?

Das ist nicht meine Meinung. Ich glaube vielmehr, daß Norden eine geradezu glänzende Intuition bewiesen hat, wenn auch die tatsächlichen Verhältnisse etwas anders liegen, als er gedacht hat.

Die Verknüpfung der Bemerkung über die Altäre der unbelkannten Götter mit den Betrachtungen über die Sittsamkeit des Hippolytos widerspricht zwar nicht dem Zusammenhang, in dem wir sie bei Philostrat finden, aber sie ist durch ihn doch nicht innerlich motiviert, und die Tatsache selbst ist so obskur, daß man nicht begreift, wie darauf die Rede als auf etwas allgemein Bekanntes kommt. Die Frömmigkeit Athens ist seit alters ein stehender Punkt des Lobes. Aber die Begründung, die hier dafür gegeben wird, daß die Athener sogar unbekannten Göttern Altäre gebaut haben, ist ein ausgesuchter Gedanke, und daß dieser hier, wo er uns zum erstenmal in der Literatur begegnet, wie ein locus communis behandelt wird, ist ein sicheres Zeichen, daß er nicht von dem Schriftsteller selbst, zum mindesten nicht an dieser Stelle, gefunden ist. Dazu kommt, daß Damis ja auch gar keinen Anspruch darauf macht, sondern ihn dem weisen Apollonios in den Mund legt.

Ferner hat Norden ohne Ziveifel darin recht, daß zwischen diesem Gedanken und dem Vortrag des Apollonios in Athen eine Verwandtschaft besteht, nur daß dies eben nicht ein wirklich, sondern nur angeblich gehaltener Vortrag ist. Wenn nun Damis den Apollonios in diesem Vortrag von der Opferfreudigkeit der Athener ausgehen ließ, so würde für diese allgemeine Behauptung die Beobachtung, daß sie sogar unbekannten Göttern Altäre bauten, eine Bestätigung und einen besonders beweiskräftigen Beleg gebildet haben.

Eine entsprechende Gedankenverbindung finden wir nun tatsächlich in der Apostelgeschichte:

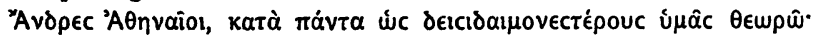

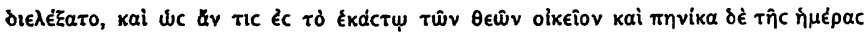

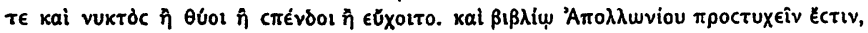

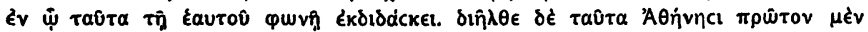

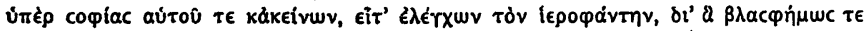

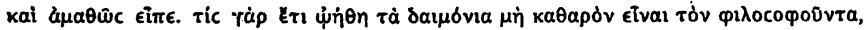

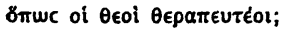




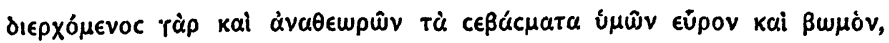

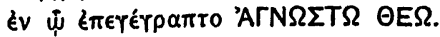

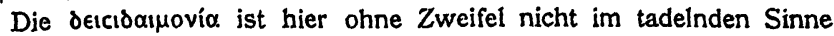
gemeint und entspricht praktisch der $\varphi \mathrm{i} 0 \theta u c i \alpha$.

Damis schob dem Apollonios noch ein zweites Motiv für seinen Vortrag unter, das Philostratos sogar an erster Stelle hervorhebt: er

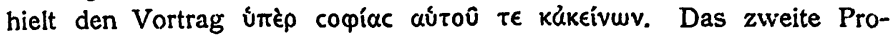
nomen kann sich nach dem Zusammenhange nur auf die Athener beziehen. Die Weisheit der Athener kann Apollonios nur in der peinlichen Gewissenhaftigkeit gefunden haben, mit der sie allen nur denkbaren. Göttern opferten. Aber des Apollonios eigene Weisheit wird Damis doch wohl noch durch etwas anderes als durch bloß äußerliche Opfervorschriften ins Licht gesetzt haben. Es ist daher sehr wahrscheinlich, daß Damis inn in dem Vortrag auch von dem höheren

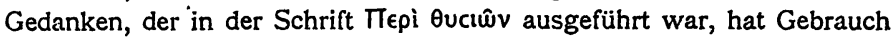
machen lassen, daß man dem großen, von allem abgesonderten und über alles erhabenen Gotte nicht opfern dürfe.

Mit diesem Gedanken aber berührt sich die Apostelgeschichte auf das engste, wenn in ihr gesagt wird, der unbekannte Gott sei der Schöpfer Himmels und der Erden, der nicht in Tempeln von Menschenhand wohne und nicht dem Werke menschlicher Kunst und Gedanken ähnlich sei, mit dem man, wie Apollonios sagte, als dem Schönsten von allem Seienden nur durch das Schönste in uns, den Geist, verkehren dürfe, der keines Werkzeugs bedarf. ${ }^{1}$

Wenn diese Konstruktion richtig ist, so hat ein Parallelismus nicht zwischen Apollonios und Lukas, wohl aber zwischen Damis und Lukas bestanden.

Wann ist der Roman des Damis geschrieben? Das weiß kein Mensch, sicher ist nur, daß er erst nach dem Tode des Nerva erschienen sein kann.

Nach den Regeln der Logik sind nun drei Fälle möglich: entweder der Parallelismus ist, um es kurz und konventionell zu sagen, zufällig, oder es besteht eine Abhängigkeit, sei es des Lukas von Damis oder des Damis von Lukas.

Eine solche Art der Erörterung wird manchem pedantisch und weitschweifig erscheinen. In der Tat ist die Abhängigkeit des christ-

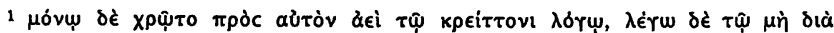

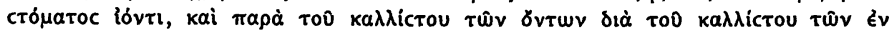

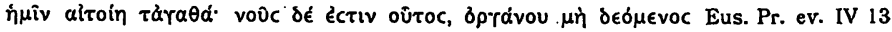

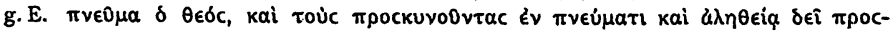
KUvยîv Joh IV 24. 
lichen Schriftstellers, der mit der Form der profanen Literatur augenscheinlich Fühlung sucht, aus allgemeinen Gründen von vornherein wahrscheinlich, und Norden hat in musterhafter Weise den literarischen Hintergrund der Areopagrede aufgehellt. Allcin wie wenig solche Darlegungen auf den Eindruck machen, der einmal von einer vorgefaßten Meinung befangen ist, zeigt Harnacks Kritik an Norden.

Ich will nun einmal die ganze eben vorgetragene Konstruktion beiseite lassen und mich nur an den überlieferten Satz von den Altären unbekannter Götter aus dem Roman des Damis in der Lobrede des Apollonios auf Timasion halten. Mag sich die Einführung dieses Satzes in die Lobrede wie immer erklären, so liegt, abgesehen von dem charakteristischen Unterschied des Numerus, auch in der Lobrede bei Damis der gleiche Gedanke wie in der Apostelgeschichte zugrunde:

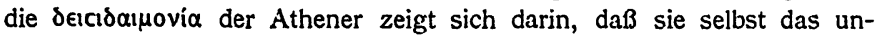
bekannte Göttliche verehren. Differenziert wird dieser Gedanke dadurch, daß Lukas eine christliche Tendenz durch den Singular hineinträgt.

Die hinter dem Gedanken stehende Tatsache wird uns nur von einem einzigen Schriftsteller, dem Periegeten Pausanias, bezeugt. Nach

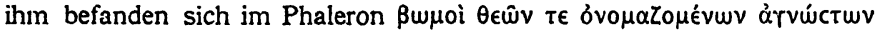
kai ì über die Streitfrage gehandelt, ob àrvwictuv sich auch auf ípúwv beziehe. . Ich bin der Meinung, daß er dies mit Recht verneint hat (S. 55 Anm. 1). Aber will Pausanias wirklich sagen, daß es im Phaleron einen Bupòc 'Hpwwv gegeben habe? Dann konnte er doch

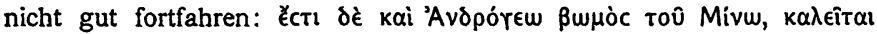
$\delta \epsilon \in$ "Howoc, denn dieser, nach Pausanias von den Kundigen dem Androgeos beigelegte Altar eines unbenannten Heros wäre doch schließlich einer von den $\beta w \mu$ oi 'Hpwww gewesen, und daß es nahe beiein-

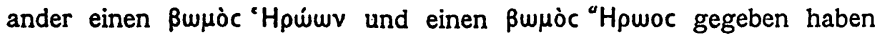

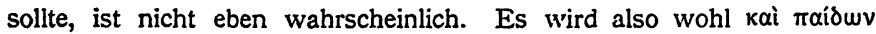

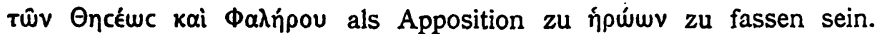
Ebensowenig aber wird man im Phaleron verschiedene Altäre unbekannter Götter nebeneinander gesehen haben, und es wird ja auch wohl von niemand bezweifelt, daß der Plural $\beta w \mu o i$ auf die vier, oder wie ich meine, drei Altäre zu verteilen ist. Aber festgestellt werden muß dies, weil auch Damis den Plural gebraucht, für den diese $\mathrm{Er}$ klärung nicht anzuwenden ist. Lukas aber mit seinem Singular $\beta w \mu$ òc entspricht der Beschreibung des Pausanias und also wohl auch der Wirklichkeit.

Generalisiert nun Damis oder gebraucht er den Plural, weil er 


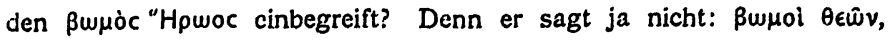

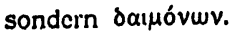

Ich brauche wohl nicht zu sagen, wie unwahrscheinlich das letztere ist und wie wenig die ganze Stelle danach aussieht, dab ihr Verfasser dabei irgendwelche Anschauung vor dem geistigen Auge hat. Dadurch bestätigt sich nun aber wieder, daß Damis hier einen ausgeprägten тónoc aus der Erinnerung verwendet.

Dagegen läßt Lukas seinen Paulus ausgesprochenermaßen aus lebendiger Anschauung schöpfen. Als er die Stadt durchwandert und die Gegenstände ihrer Verehrung mustert, findet er auch den Altar mit der Aufschrift: Dem unbekannten Gott.

Allein ich fürchte, der Schein trügt. Ich vermag dieses Interesse für die Sehenswürdigkeiten Athens so wenig wie Norden (S. 33) mit dem Eindruck, den die Persönlichkeit des großen Apostels uns in seinen Briefen hinterlassen hat, $z u$ vereinigen; doch ich will solche Gefühlswerte ebensowenig wie Norden geltend machen. Aber ist es wahr-

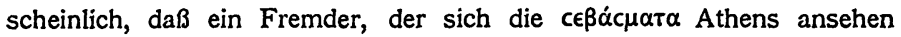
will, zu diesem Ende in das Phaleron hinabschlendert und dort die Inschriften auf unbedeutenden und unbeachteten Monumenten studiert? Macht es übrigens den Eindruck, daß der Areopagredner sich bewußt ist, daß der Altar nicht mitten in der Stadt, sondern in der Hafenstadt verborgen lag, wo er unter den Fremden nur bei Leuten wie Pausanias, die auf die Anomalien des Kultus ganz besonders zu achten gewohnt waren, ein Interesse erregte? Und ist es eine natürliche Redewendung oder ausgesuchte Rhetorik, daß all den herrlichen $c \in \beta a ́ c \mu \alpha \tau \alpha$ der Athener der eine kleine erbärmliche Altar des unbekannten Gottes entgegengestellt wird? Man sollte meinen, wenn Paulus selbst eine solche Entdeciung gemacht hätte, so würde er etwas mehr natürliches Erstaunen in seine Worte gelegt und die Tatsache nicht, wie es in der Areopagrede geschieht, als etwas Selbstverständliches hingestellt haben.

Allein Paulus möge irgendeinem Zufall die Entdeckung zu verdanken gehabt haben, und Lukas möge die Verantwortung für den rhetorischen Aufputz tragen. Ist das so, so hat sich Paulus einer Fälschung schuldig gemacht, und diese pia fraus traue ich wohl dem Verfasser der Apostelgeschichte, nicht aber dem Apostel selber zu.

Aber darüber will ich mit niemand streiten. Ich will einzig und allein die Frage im Auge behalten, ob wir es im Anfang der Areopagrede mit einem literarischen und also entlehnten Motiv oder mit einem aus der Kenntnis der Wirklichkeit entsprungenen selbständigen Gedanken $z u$ tun haben. 
Für den ersten Teil der Alternative hat Norden aus seiner reichen Belesenheit so viel beigebracht, daß es für viele überzeugend sein wird. Aber wer die Dinge mit bloßer Dialektik meistern will, wird sich damit nicht zufriedengeben. Diese aber werden nicht umhin können, die Forderung als berechtigt anzuerkennen, daß dann auch die äußeren Umstände, in welche die Rede eingekleidet ist, mit den tatsächlichen Verhältnissen übereinstimmen müssen.

Daß Paulus zuerst in der Synagoge auftritt $(17,17)$, entspricht seiner Gewohnheit. Daß er aber dann ohne weiteres auch auf dem Markte mit den gerade Anwesenden zu disputieren anfängt, ist auffällig. Das Motiv dazu muß man in der Erregung sehen, die ihn bei dem Anblick der götzendienerischen Stadt packt $(17,16)$. Zu dieser Erregung stimmt aber schlecht die äußerst diplomatische Anlage der Areopagrede.

Wie aber kommt Paulus auf den Areopag? Man packt ihn und

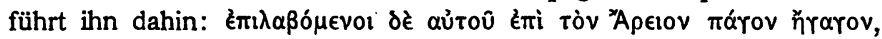
also wie einen Angeklagten, der sich verantworten soll. Offenbar ist die Veranlassung dazu die Behauptung, er sei ein Verkünder fremder

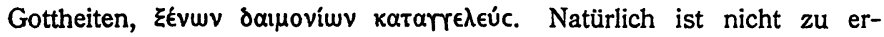
warten, daß, wenn eine solche Beschuldigung erhoben wurde, nun ein

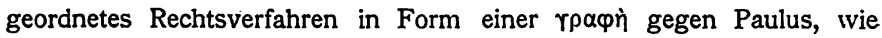
seinerzeit gegen Sokrates, eröffnet worden wäre, da Paulus ja kein athenischer Bürger war. Es ist allenfalls denkbar, daß eine ảmarwrì erfolgt wäre, als gegen einen auf der Tat ertappten Gotteslästerer und Aufrührer. Aber dann wäre er zunächst ins Gefängnis geführt und nicht vor einen Gerichtshof, geschweige denn den Areopag.

Nun führt aber der Berichterstatter den Ansatz, den er nimmt, gar nicht durch, und Paulus hält seine Rede, ohne daß von Richtern oder von einem Urteil die Rede ist, und dieselben Leute, die ihn auf den Areopag bringen, sind es auch, die an ihn die Frage richten, was es mit seiner neuen Lehre auf sich hat, obwohl sie doch darüber den ganzen Tag mit ihm auf dem Markte disputiert haben. Aber wenn der Areopagite Dionysios als der einzige von den wenigen Männern, die gläubig werden, genannt wird, so muß man doch wohl annehmen, $\mathrm{daB}$ er in seiner Eigenschaft als Areopagite bei der Rede zugegen war. Dagegen wird der Gedanke wieder auf einen allgemeinen Versammlungsplatz gelenkt, wenn es heißt, Paulus sei mitten auf den Areshügel getreten. Einen solchen, viele Zuhörer fassenden Platz, wie es doch offenbar gedacht ist, gab es auf dem Areshügel gar nicht.

Es fehlt auch hier jede lebendige Anschauung, und offenbar kämpft der Schriftsteller mit zwei verschiedenen Konzeptionen, die er nicht 
zum Ausglcich miteinander $\mathrm{zu}$ bringen vermocht hat. Dabei ist aber nicht zu verkennen, daß er von dem Gedanken beherrscht ist, religiöse Streitigkeiten gehörten in Athen vor das Forum des Areopags. Zudem

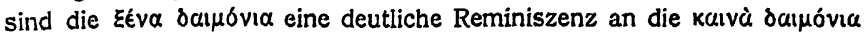
in dor Anklageschrift gegen Sokrates, und es ist daher sehr wahrscheinlich, daß Lukas angenommen hat, der Prozeß des Sokrates habe sich auf dem Areopag abgespielt, um so mehr als wir diese Meinung sogar bei Origenes finden (C. Celsum IV 67 und V 21).

In der Tat muß der Areopag in späterer Zeit mit Prozessen wegen Gottlosigkeit befaßt worden sein, denn wir haben wohl nicht das Recht, die Angaben des Diogenes Laertius anzufechten, daß der Philosoph Theodoros wegen einer respektlosen Bemerkung gegen den Hierophanten Eurykleides beinah vor den Areopag gekommen wäre, wenn ihn nicht Demetrius, der Phalereer, gerettet hätte (II 101), ${ }^{1}$ und Stilpon wegen Asebie wirklich von den Areopagiten verbannt sei (II 116), wenn auch die Details einen anekdotenhaften Charakter tragen. ${ }^{2}$ Dazu

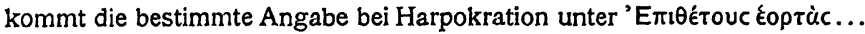

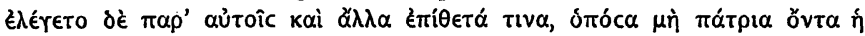

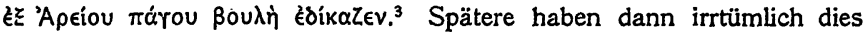
Rechtsverfahren auch auf die frühere Zeit übertragen, wie Origenes bei Sokrates und Clemens Alexandrinus bei Aeschylus (Strom. II 14, 60).

Mit Recht hat man sich auch gewundert, daß Lukas Stoikern und Epikureern das gleiche Interesse für die Lehre des Paulus beilegt. Allerdings versucht er sie verschieden $\mathrm{zu}$ charakterisieren, denn das verächtliche Urteil $\tau i$ ồv $\theta \in$ é wohl von den Epikureern kommen. Aber sie scheinen doch mit den Stoikern in der Denunziation einig zu sein, und das zeigt eine sehr oberflächliche Kenntnis der Dinge.

Bezeichnend ist auch die Art, wie die landläufige Charakteristik der Athener durch die Anwendung des Imperfektums (nủkaipouv) zu einer persönlichen Beobachtung des Berichterstatters gestempelt wird.

Wenn man sich diese ganze Aufmachung überlegt, so sieht man, mit was für einem Manne man es $z u$ tun hat. Das ist kein Begleiter des Paulus, der aus lebendiger persönlicher Erfahrung schreibt, auch keiner, der. einen solchen Bericht nacherzählt, sondern jemand, der weder eine Anschauung des Ortes, noch eine am Ort erworbene

1 Nach anderer Angabe wurde er zum Schierlingsbecher verurteilt.

2 Vgl. J. H. Lipsius, Das attische Recht und Rechtsverfahren, 1908, S. 367.

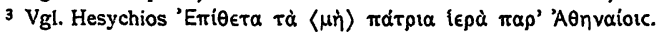


Kenntnis der Verhältnisse hat; ein nicht ungewvandter Schriftsteller, mit einer ganz oberflächlichen Bildung, die absichtsvoll hervorgekehrt wird und doch ganz auf unsicheren literarischen Reminiszenzen beruht. Das ganze, absichtlich aufgetragene lokale Kolorit macht die Erzählung nicht wahrscheinlicher, sondern verrät vielmehr ihren wirklichen Charakter. Ist aber die Einkleidung der Rede literarisch bedingt, so ist es diese auch, und die Bemerkung über den Altar des unbekannten Gottes kann dann nicht von der Wirklichkeit ausgegangen sein. Dann aber kann die Änderung des Plurals in den Singular ungleich milder beurteilt werden.

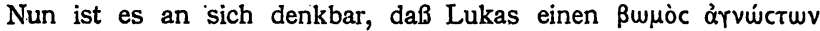
$\theta \epsilon \omega \hat{v}$ irgendwo erwähnt gefunden hatte und dann in der Rede seine Betrachtung daran knüpfte. .

Allein erstens ist es nicht gerade wahrscheinlich, daß Lukas in der topographischen Literatur sich umgetan habe. Sodann konnte

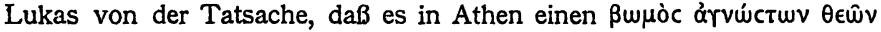
gab, ohne einen $Z$ wischengedanken, wie wir ihn oben auch für die Lobrede auf Timasion angenommen haben, daß nämlich Verehrung unbekannter Götter ein Zeichen von Frömmigkeit sei, gar nicht auf die Annahme einer latenten Verehrung des Christengottes bei den Athenern kommen. Dieser $Z$ wischengedanke aber mußte einem Christen ganz fern liegen. Eine Fülle von ărvwctor $\theta \in$ ei konnte ihm nur schauderndes Entsetzen erregen.

Etwas anderes war es, wenn er die Behauptung auf heidnischer Seite ausgesprochen fand, Verehrung unbekannter Götter sei ein Beweis von Gottesfurcht. Eine solche Behauptung forderte geradezu zur Berichtigung heraus und diese Berichtigung lieferte dann von selbst das Sprungbrett für einen Anlauf zu einem monotheistischen Beweise.

Es wäre noch zu fragen, ob Lukas bei der Umsetzung des Plurals in den Singular an einen ihm gegebenen und geläufigen Begriff angeknüpft habe. Das ist Nordens Meinung (S. 58) und seine gelehrten und zum großen Teil ganz neuen Ausführungen über den ărvwctoc $\theta$ eòc in der gnostischen Literatur bilden ohne Zweifel ein ganz besonders interessantes Kapitel seines Buches. Für die Berveisführung, $\mathrm{da} B$ dieser Begriff vorchristlich sei, ist seine Interpretation des Logion Mt 11, 25-30 entscheidend, zu deren selbständiger Nachprüfung ich zu meinem Bedauern immer noch keine Zeit gefunden habe. Wie es nun aber auch mit dem Alter des gnostischen Begriffes stehe und ob er Lukas geläufig gewesen sein möge oder nicht, so halte ich eine Beziehung des Lukas darauf schon durch die Erkenntnis ausge- 
schlossen, daß ihm der Grundgedanke von heidnischer Seite gegeben war. Jede Erinnerung an die Gnostiker hätte seine Konzeption nur trüben können. Er nimmt aber das Wort đ̈rvwctoc ganz offenbar in demselben vulgären Sinne, in dem es auch bei Philostratos steht. Er macht auch der arvwcía keine Konzession (S.129). Die liegt nur in der Form, und in der Form hat auch Paulus nach seinem eigenen Bekenntnis (1 Kor 9, 20 f.) Heiden wie Juden sich anzupassen nicht verschmäht. Lukas ist durchaus der Ansicht des Paulus, daß die Zeit der Unwissenheit vorüber ist, da nun der Zorn Gottes vom Himmel herab enthüllt wird (Rom 1,18). Das Bekenntnis aber zu einem Grvwcтос $\theta \in \delta$ c, das Lukas den Athenern beilegt, schließt die Verehrung des Unsichtbaren in dem sichtbaren Gleichnis eines vergänglichen Menschen, die der Areopagredner nicht minder als der echte Paulus verdammt, begrifflich aus, und darum bildet sie einen so glücklichen Anknüpfungspunkt für die christliche Anschauung.

Ist nun rein in abstracto bewiesen, daß der Gedanke, mit dem Lukas die Areopagrede einleitet, auf einer Entlehnung von heidnischer Seite beruht, so gewinnen Nordens konkrete Kombinationen in der von mir modifizierten Form einen Grad von Wahrscheinlichkeit, der, wie mir scheint, an Gewißheit grenzt.

Das einzige, was, soweit ich sehe, Bedenken erwecken kann und ohne Zweifel bei vielen Widerspruch dagegen herausfordern wird, ist die Tatsache, daß der Roman des Damis nicht vor dem Anfang des zweiten Jahrhunderts entstanden sein kann und die Möglichkeit zugegeben werden muß, daß er noch beträchtlich jünger war.

Allein das letztere ist eine Annahme, die auch an und für sich durchaus keine größere Wahrscheinlichkeit hat.

Der Ninevite Damis, mit dem Apollonios auf seiner Reise nach Indien in dem alten Ninive zusammenstößt, an dessen verlassenen Trümmern schon Xenophon ohne eine Ahnung ihrer wirklichen historischen Bedeutung vorbeimarschierte, ist eine so handgreifliche Fiktion, daß sie sich beinah absichtlich $z u$ verraten scheint. Ebendarum kann man seine angeblichen Selbsterlebnisse nicht gut einen Schwindel nennen. Jedenfalls darf man nicht sagen, hier hat Pseudodamis geschwindelt, dort hat er nicht geschwindelt, weil das Detail seiner Angaben zu erlesen ist (S. 39).' Seine Darstellung ist überall von derselben Farbe überzogen und das etwa hier und da zugrunde liegende Tatsächliche dürfte schwer mit Sicherheit zu erkennen sein. Es ist sehr wohl möglich, daß Apollonios einmal einen Streit mit einem Hierophanten in Athen gehabt hat, der ihm die Weihen verweigerte. 
Aber ebensogut kann das eine reine Erfindung sein. Dafür spricht der Umstand, daß dieser Streit in dem Roman ein nicht allzu geschicktes Mittel bildet, einen Zusammenhang zwischen einem doppelten Aufenthalt des Apollonios in Athen in der Weise herzustellen, daß dadurch seine Weissagungsgabe in helles Licht gesetzt wird. Außerdem dient er, wie schon angegeben, zur Einführung eines religionswissenschaftlichen Vortrags, von dem es sicher ist, daß er jedenfalls nicht so gehalten ist. $\mathrm{Da}$ diesem angeblichen Vortrage eine echte Schrift des Apollonios von Damis zugrunde gelegt wòrden war, so ist die Möglichkeit zuzugeben, daß schon in ihr die Verknüpfung der Opferfreudigkeit der Athener mit ihrem Kult der unbekannten Götter enthalten war. Aber für die Apostelgeschichte hat das keine Bedeutung.

Dagegen ist bemerkenswert, wie der Roman eingeleitet war. Damis traf den Apollonios in Ninive an einem nach Barbarenart errichteten Götterbilde, das aber die Io darstellte und von dem Apollonios besser Bescheid zu geben wußte als die Priester und Propheten (Philostr. I 19). Auf einen Fall, in welchem die Reisenden am Hyphasis Altäre mit Aufschriften finden, die mitgeteilt werden (I 43), ${ }^{1}$ auf einen andern, wo Apollonios in der Troas ein verschüttetes Bild mit der Aufschrift: Dem göttlichen Palamedes, entdeckt (IV 13), hat Norden bereits hingewiesen (S. 41 Anın. 2). Man kann vielleicht noch III 25, wo ein Bild des

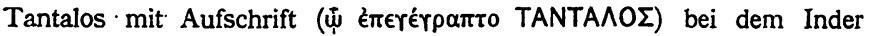
Iarchas beschrieben wird, und. II 9, wo sich ein silberner Schild in Pytho mit einer Weihinschrift des Dionysos an den delphischen Apollon erwähnt findet, hinzufügen. So zeigt sich mit der Aufspürung des athenischen Kultuskuriosums die Schilderung, die der Roman von dem Wesen des priesterlichen Pythagoreers gibt, im besten Einklang.

Der Roman war durchaus auf Unterhaltung und Erbauung und Erregung eines vermeintlich philosophischen Interesses angelegt und muß in der Beziehung Verwandtschaft mit Büchern wie der Abaris des Herakleides und der Lykon des Ariston gehabt haben. ${ }^{2}$ Der Schwindel beginnt erst mit Philostratos, der ihn als Geschichte behandelt, obwohl auch das nur Maske ist und es ihm in Wahrheit darauf ankommt, das rein Unterhaltende noch mehr zur Geltung zu bringen.

Merkwürdig ist, daß Philostratos so tut, als sei das Werk erst zu seiner Zeit ans Licht getreten (I 3). Das ist ganz gewiß nur

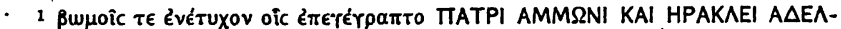
$\Phi \Omega 1$ usw.

2 Vgl. Plutarchs Bemerkungen in der Einleitung zu De audiendis poetis, Mor. p.14 E. Zeitschr. f. d. neutest. Wiss. XIV. Jahrg. 1913. 
literarische Einkleidung. Am allerwenigsten darf man daraus schliefen, Philostrat habe ein solches Buch gar nicht in Händen gehabt, sondern selbst erfunden. Um das widerlegt $\mathrm{zu}$ sehen, braucht man nur, wie Norden treffend bemerkt (S. 37 Anm. 1), Philostrat selbst $z u$ lesen. Philostrats Hauptquellen waren nach seiner eigenen Angabe ein Buch des Maximus von Aegae über Apollonios' Aufenthalt in Aegae und die Papiere des angeblichen Damis (I 3). Die erste Quelle reicht bis I19, dann beginnt die zweite. Sofort merkt man, daß hier ein anderer Geist weht, und Philostratos müßte ein einzigat tiger Stilkünstler gewesen sein, wenn er rein erdichtete Quellen derartig zu differenzieren verstanden hätte.

Es ist aber durchaus glaubwürdig, daß die Aufzeichnungen des Pseudodamis aus der unmittelbaren Umgebung des Apollonios hervorgegangen und bald nach dessen Tode erschienen sind. Gerade wenn es ein altes und schon beinah vergessenes Werk war, ist es verständlicher, daß Philostratos sich zu einer Neubearbeitung entschloß und es mit dem Glanze seines eigenen Stiles durchleuchtete.

In gewissem Sinne kann man zu den Vorgängern des Pseudodamis auch den Verfasser der'Kyrupädie rechnen, in Absicht auf die erzieherische Tendenz ganz sicher und vielleicht auch bis zu einem gewissen Grade hinsichtlich des Verhältnisses zwischen Verfasser und Stoff.

Die erzieherische Tendenz in dem Roman des Damis ist unverkennbar und wird an dem angeblichen Verfasser selbst entwickelt, der durch den Umgang mit Apollonios sich zu einem Philosophen ausbildet. Wie aber Xenophon zu der Darstellung seines Ideales eines Fürsten und Menschen und zu der Projektion dieses Ideales auf einen angeblich historischen und tatsächlich doch ganz phantastischen Hintergrund durch seinen kurzen und durchaus nicht intimen Verkehr mit dem Prinzen Kyros begeistert wurde, so ist es sehr wohl möglich, ja beinah wahrscheinlich, daß eine unmittelbare Berührung zwischen Pseudodamis und Apollonios noch stattgefunden hat.

Wie wenig unsere Apostelgeschichte, eine sehr wohl überlegte und trotz mancher Züge aus dem Bereich des Wunderbaren keineswegs von unbezwinglicher Lust am Fabulieren getragene, sondern von einem rationalistisch veranlagten Geiste beherrschte Darstellung, mit dem Apolloniosroman des Damis innerlich zu tun hat, braucht nicht gesagt zu werden. Sehr viel näher standen ihm die apokryphen Apostelgeschichten in ihrer alten ursprünglichen Form, nur daß sie aus der Atmosphäre einer ungleich stärkeren geistigen Fäulnis stammten. Der 
Verfasser der Apostelgeschichte hat eben nur ein einzelnes Motiv aus dem Roman in kühner Umbildung so wirkungsvoll benutzt, dab er damit selbst den bedeutendsten Theologen unserer Zeit in seinen Bann gezogen hat, den auch meine Ausführungen gewiß nicht brechen werden. Wenn aber durch diese die Entstehungszeit der Apostelgeschichte bis etwa in den Anfang der hadrianischen Zeit hinabgerückt wird, so würde mir dazu der ganze Charakter der Schrift zu stimmen scheinen, und ich würde das Ergebnis meiner auf diesen Punkt gar nicht gerichteten Untersuchung auch nach dieser Seite für einen Gewinn halten. Sollte mir aber mein Versuch, Nordens Auffassung in einem Punkte zu berichtigen, gelungen sein, so bin ich mir wohl bewußt, daß dies nur ein Nebenpunkt ist, der die Hauptsache unberührt läßt. 